\title{
Contrast vision and optic neuritis: neural blurring
}

\author{
ROBERT F HESS \\ From the Physiological Laboratory, University of Cambridge, and the Department of Neurology, \\ Neurosurgery and Psychiatry, Addenbrookes Hospital, Cambridge, UK
}

SUMMARY In order to understand the vision of patients with long standing optic neuritis, contrast perception was analysed in some detail for three patients with uniocular anomalies. Contrast detection and contrast matching experiments each demonstrate anomalies in contrast processing for eyes with optic neuritis. In this regard, optic neuritis differs from all other visual anomalies so far investigated where the suprathreshold deficits (from matching experiments) are slight compared with those measured under threshold conditions (from detection experiments). Contrast discrimination is also impaired to a mild degree in optic neuritis but this cannot be predicted on the basis of the matching results. These results bear upon the currently proposed models for contrast coding in the normal visual system as well as the nature of the underlying pathology in optic neuritis.

Over the past decade our concept of the type of visual loss that occurs in patients with optic neuritis has been greatly clarified by the measurement of contrast thresholds with sinusoidal gratings of different bar width or spatial frequency. Following from the original work of Bodis-Wollner ${ }^{1}$ on cerebral lesions, other workers ${ }^{23}$ have shown that not only can thresholds for low spatial frequency stimuli be affected independently of acuity but thresholds also can be raised for only a limited band of intermediate spatial frequencies.

Although this threshold information is interesting for reasons of classification and diagnosis it fails to answer questions relating to the visual perception of these patients because it does not directly assess the perception and processing of suprathreshold contrast information of which everyday images are composed. The fact that threshold and suprathreshold contrast processing can differ is evident for normal vision ${ }^{5}$ and amplified in the case of the developmental anomalies (amblyopia) of vision. ${ }^{6}$ In each case (normal or amblyopic) conditions can be found for which thresholds are raised yet suprathreshold performance is normal.

In order to gain more insight into the visual perception of patients with optic neuritis and not just their abilities for threshold detection, contrast pro-

\footnotetext{
Address for reprint requests: Dr RF Hess, Dept of Physiology, Cambridge CB2 3EG, UK.
}

Received 17 March 1983 and in revised form 14 May 1983. Accepted 2 June 1983 cessing above threshold was investigated with two different but complimentary techniques. First, contrast matching was measured between the normal and anomalous eye of patients with optic neuritis. Secondly, incremental contrast discrimination was examined over a wide range of stimulus contrast for normal and anomalous eyes.

We have sought a better understanding of the vision and thus the visual defect in optic neuritis for stimuli whose contrast is at and above threshold. We relate these findings in optic neuritis to each of the two current psychophysical models of how contrast is processed in the normal visual system. ${ }^{45}$ To this extent, this report differs in its approach from that of Sjostrand and Abrahamsson ${ }^{7}$ who reported on the recovery of suprathreshold contrast perception in optic neuritis. We have concentrated on the remaining deficit rather than the recovery that characterises the initial attacks. We have used more established uniocular cases and examined in more detail contrasts just suprathreshold. The table gives the clinical details of the patients studied.

\footnotetext{
Methods

Apparatus

Vertical sinewave gratings (temporal frequency $=\mathrm{OHz}$ ) were generated analogically or digitally and displayed on each half of a specially constructed $20 \mathrm{~cm} \times 30 \mathrm{~cm}$ video display (P31 phosphor). The spatial frequency and contrast of each of these waveforms could be independently varied. Contrast was linearly related to input voltage up to $90 \%$ contrast. The mean luminance of each was equal and set to
} 
Table Clinical details of patients studied

\begin{tabular}{|c|c|c|c|c|c|c|c|c|c|}
\hline Patient & $\begin{array}{l}\text { Age } \\
(y r)\end{array}$ & Diagnosis & $\begin{array}{l}\text { Length of } \\
\text { history } \\
\text { (months) }\end{array}$ & $\begin{array}{l}\text { Visual } \\
\text { acuity }\end{array}$ & $\begin{array}{l}\text { Ishihara } \\
\text { test no } \\
\text { of errors }\end{array}$ & $R A P D$ & $\begin{array}{l}\text { Optic } \\
\text { disc }\end{array}$ & $\begin{array}{l}\text { Visual } \\
\text { field }\end{array}$ & $\begin{array}{l}\text { Contrast } \\
\text { threshold } \\
\text { loss }\end{array}$ \\
\hline KT & 34 & $\begin{array}{l}\text { Unilateral optic } \\
\text { neuritis }\end{array}$ & 6 & $6 / 24$ & None & No & Normal & Normal & $\begin{array}{l}\text { Localised } \\
\text { mid-frequency } \\
\text { loss }\end{array}$ \\
\hline MM & 49 & $\begin{array}{l}\text { Unilateral optic } \\
\text { neuritis from } \\
\text { multiple sclerosis }\end{array}$ & $\begin{array}{l}12 \\
\text { (visual) }\end{array}$ & $6 / 60$ & 7 & Yes & Pallor & $\begin{array}{l}\text { Central } \\
\text { scotoma }\end{array}$ & $\begin{array}{l}\text { Mainly } \\
\text { mid-frequency } \\
\text { loss }\end{array}$ \\
\hline JL & 46 & $\begin{array}{l}\text { Unilateral optic } \\
\text { neuritis }\end{array}$ & 18 & $6 / 6$ & 1 & No & Pallor & Normal & Low pass loss \\
\hline SS & 28 & $\begin{array}{l}\text { Unilateral optic } \\
\text { neuritis }\end{array}$ & 24 & $6 / 12$ & 6 & Yes & Pallor & Normal & $\begin{array}{l}\text { Generalised } \\
\text { spatial loss }\end{array}$ \\
\hline IW & 20 & $\begin{array}{l}\text { Bilateral optic } \\
\text { neuritis from } \\
\text { multiple sclerosis }\end{array}$ & $\begin{array}{l}24 \\
\text { (visual) }\end{array}$ & $\begin{array}{l}6 / 60 \\
R \& L\end{array}$ & $\begin{array}{l}10 / 4 \\
\mathrm{R} / \mathrm{L}\end{array}$ & Yes & $\begin{array}{l}\text { Bilateral } \\
\text { pallor }\end{array}$ & $\begin{array}{l}\mathbf{R} \& \mathbf{L} \\
\text { central } \\
\text { scotoma }\end{array}$ & $\begin{array}{l}\text { Generalised } \\
\text { spatial loss }\end{array}$ \\
\hline
\end{tabular}

$200 \mathrm{~cd} / \mathrm{m}^{2}$. The subject viewed these gratings from a distance of $114 \mathrm{~cm}\left(10^{\circ}\right.$ field size) such that, by the use of a partition, the gratings were seen dichoptically. A slowly rotating sectored disc alternately occluded one eye and simultaneously presented a grating to the other eye every second. Furthermore, the gratings were presented for an 8 second interval every 20 seconds in this mode. This switching from grating to mean luminance was used to avoid differential adaptation effects at higher contrasts. ${ }^{8}$ The subject either made a series of threshold settings or suprathreshold matches during these exposures. A tone signalled the beginning of the 8 second test period.

The method of adjustment $(\mathrm{N}=5)$ was used for all matches. At first the subject was carefully refracted for ametropia and a viewing distance of 1 metre. The spatial frequencies were then subjectively matched for normal and anomalous eyes. Contrast thresholds were then measured under the stimulus conditions already discussed and contrast matches at $3 \mathrm{~dB}$ steps (in random order) were then made up to $90 \%$ contrast for the two objectively equal spatial frequencies. A grating of present contrast (referred to as the standard) was always presented to the anomalous eye. Racalibrated Kodak neutral density filters in goggles were used to reduce the luminance (of both fields) and angular field size was varied by changing distance. All results on the patients used were rechecked on at least one subsequent occasion.

\section{Contrast discrimination experiments}

These experiments involved a successive, two alternative forced choice procedure for both the measurement of contrast threshold and the contrast necessary to determine that two suprathreshold gratings were different in contrast. In the first procedure the subject was presented (threshold) with two intervals each of 500 milliseconds (time Gaussian), one of which contained a sinewave grating. The Quest psychophysical procedure was used ${ }^{9}$ which involved a staircase method driven from maximum likelihood estimates. Sixty trials were run for each threshold estimate.

In the contrast discrimination experiments the same procedure was used except that two gratings of the same spatial frequency were presented and the task was to choose which interval contained the higher contrast grating. A minimum of 60 trials were run for each condition. A PDP $11 / 20$ computer presented the stimuli and collected responses. The normal and fellow anomalous eyes were always alternately tested at each condition. The experiments were always repeated on another occasion.

\section{Results}

\section{A CONTRAST MATCHING}

In fig 1 the results for a normal inexperienced observer are shown for equating the contrast of several spatial frequencies between eyes. Each datum represents the average of five settings with vertical lines indicating \pm 1 standard deviation of the settings. In a number of cases this was less than the symbol size used. It is evident that for spatial frequencies between 0.3 and $10 \mathrm{c} / \mathrm{deg}$ equating the contrast of identical spatial stimuli each presented to a different eye can be performed in a proportional (fitting a $45^{\circ}$ line) and accurate manner (small SD).

Results for three of the cases of optic neuritis studied are displayed in figs $2-4$. In fig 2 contrast matching was performed between the normal and fellow affected eye of patient (JL) (unilateral deficit). The threshold contrast loss which is shown in the figure inset is seen not to vary with spatial frequency. When contrast was equated between the eyes of this patient at three different spatial frequencies, functions were obtained each having slope but displaced by differing amounts from the normal matching line (solid line). The amount of this vertical displacement equalled the threshold deficit (half filled symbols on these functions are threshold estimates). Such a displacement represents a constant ratio change in perceived contrast by the anomalous eye. For the $4 \mathrm{c} / \mathrm{deg}$ stimulus the perceived luminance and colour were adjusted to be equal between eyes $(\nabla)$ and this is seen not to affect the perceived contrast (circles).

Fig 3 shows results for a similar experiment conducted on another case of unilateral optic neuritis having a specific mid-frequency defect in threshold sensitivity (KT) of the type first reported by Regan, 


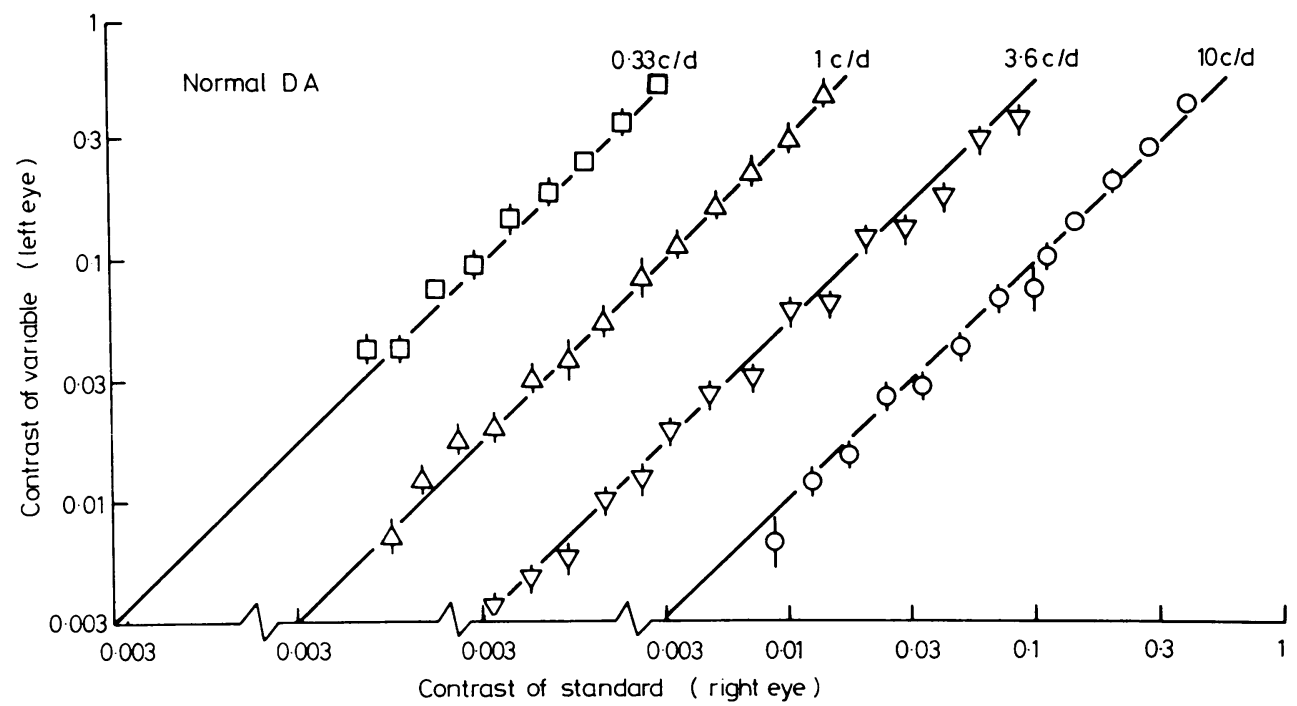

Fig 1 Contrast matching results are displayed for a normal observer for a range of spatial frequencies. $A$ standard contrast was presented to the right eye and matched by varying the contrast of an identical pattern shown to the left eye. For normal eyes in optimal focus, contrast can be matched accurately. Vertical bars indicate \pm one standard deviation.

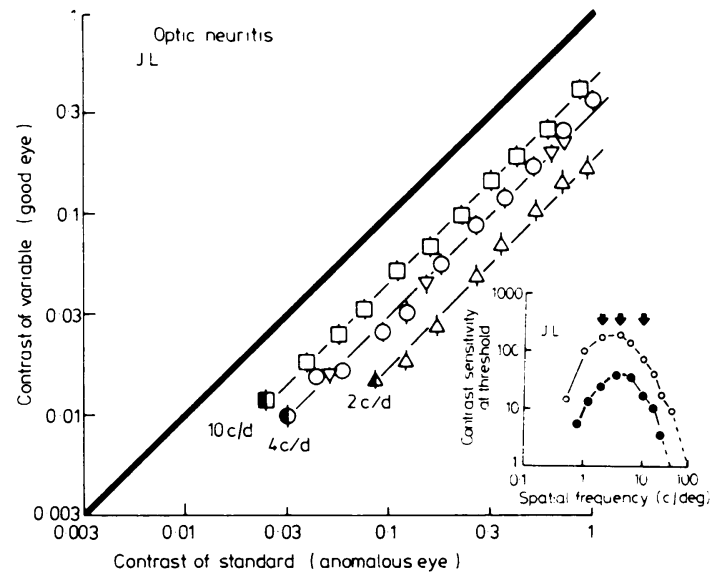

Fig 2 Contrast matching results for a case of unilateral optic neuritis (JL). Three spatial frequencies were investigated. For each frequency, a standard grating was presented to the anomalous eye at each of a number of base contrasts. An identical but variable stimulus was presented to the fellow normal eye and equated for contrast. The scales are logarithmic and the half filled symbols represent detection thresholds for normal and anomalous eyes for each stimulus. For the 4 c/deg stimulus the luminance and colour differences perceived by the anomalous eye were carefully eliminated. This does not affect the resultant contrast match. Results for a normal observer would lie along the heavy diagonal line. Inset in the figure are the detection threshold results (low pass loss) with the three spatial frequencies investigated marked by arrows. Vertical error bars indicate $\pm 1 S D$. In all cases a parallel displacement occurs in suprathreshold matching.

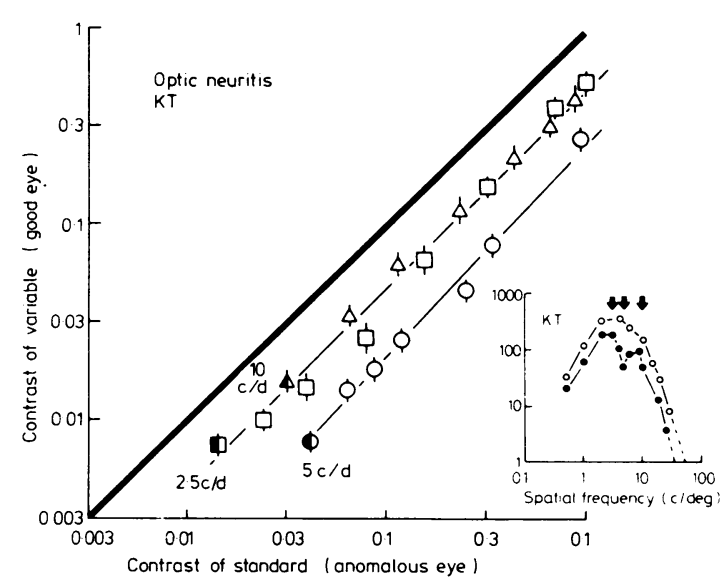

Fig 3 Contrast matching results for a case of unilateral optic neuritis $(K T)$. Three spatial frequencies were investigated. For each, a standard grating was presented to the anomalous eye at each of a number of base contrasts. An identical target but variable contrast was presented to the fellow normal eye and matched to the standard. The scales are logarithmic and the half filled symbols are detection thresholds. Results for a normal observer would fit along the sloping diagonal line. Inset in the figure are the detection threshold results (band pass loss) with the spatial frequencies position of the three gratings used in the matching experiment indicated. Spatial stimuli were chosen to correspond with the pit and shoulders of the notch loss in detection sensitivity. Vertical error bars represent $\pm 1 S D$. A parallel shift in suprathreshold sensitivity is observed for all frequencies. 


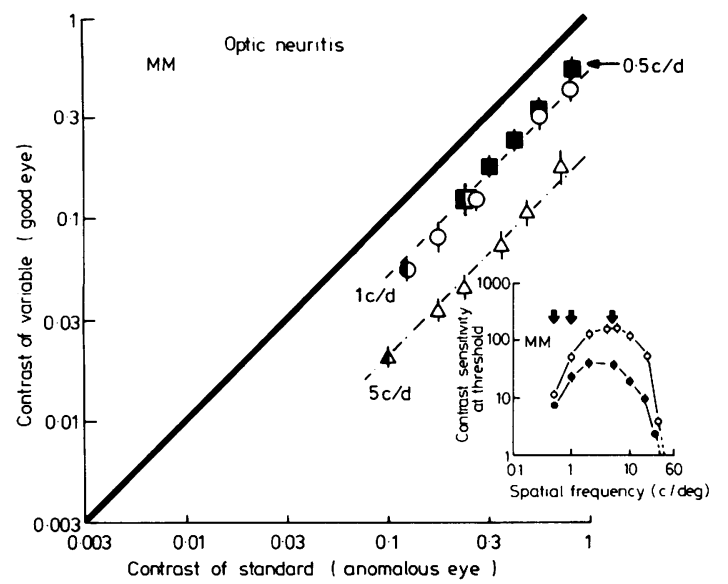

Fig 4 Contrast matching results for a case of unilateral optic neuritis (MM). Three spatial frequencies were investigated. For each, a standard grating was presented to the anomalous eye at each of a number of fixed contrasts. An identical but variable (contrast) stimulus was presented to the fellow normal eye and equated for contrast. The scales are logarithmic and the half filled symbols represent detection thresholds. Results for a normal observer would lie along the heavy diagonal line. A parallel shift of the data is seen to occur at each spatial frequency. Inset in the figure are the detection threshold results (overall broad band loss) with the spatial frequencies used for matching indicated. Vertical eror bars represent $\pm 1 S D$.
Silver and Murray. ${ }^{2}$ In this case, contrast thresholds were evenly displaced in one eye for all spatial frequencies higher and lower than the threshold notch loss (centred at $5 \mathrm{c} / \mathrm{deg}$ ). Similar results are seen for targets whose spatial frequency represent the shoulders of the threshold notch loss and a frequency representing the pit of the threshold notch loss. Results similar to those seen in fig 2 are displayed for another unilateral optic neuritis for three different spatial frequencies (fig 4).

In fig 5 the characteristic loss in contrast perception (parallel displacement) is seen to occur independently of field size. As the field size is extended from $2 \times 2^{\circ}$ to $16 \times 16^{\circ}$, the overall deficit diminishes but the form of the contrast loss remains unchanged. Similarly as luminance is reduced by interposing a neutral density filter before both normal and fellow anomalous eyes, threshold and suprathreshold function is seen also to remain unchanged in form but with a diminished contrast range (fig 6).

\section{B CONTRAST DISCRIMINATION}

The results of comparing contrast discrimination between normal eyes and those suffering from optic neuritis are displayed in figs 7 and 8. In fig 7, observer SS exhibited a contrast deficit for only low spatial frequencies $(<1 \mathrm{c} / \mathrm{deg})$. In each of the figures the contrast thresholds for normal and affected eyes is indicated by unfilled and filled arrows respectively. These thresholds were measured using an

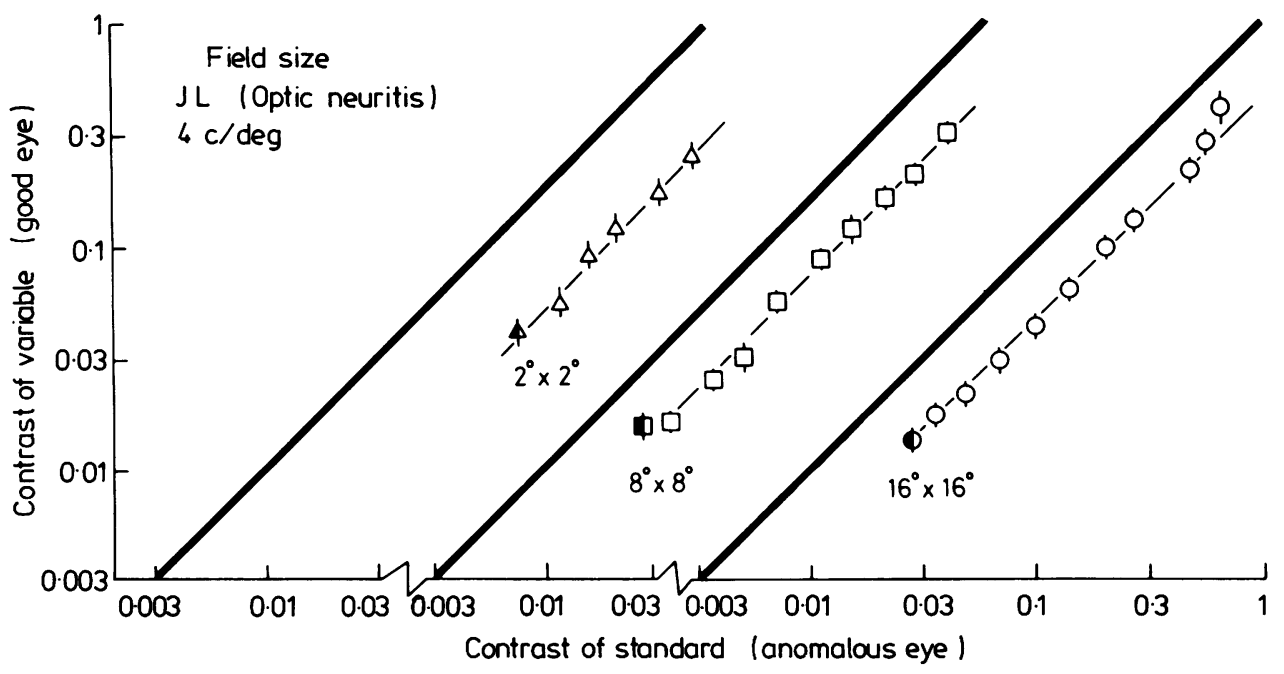

Fig 5 Contrast thresholds (half filled symbols) and suprathreshold matches (open symbols) are displayed for a uniocular case of optic neuritis. The characteristic form of the suprathreshold loss (parallel shift of unity slope) is independent of the field size of the grating stimulus out to $16^{\circ} \times 16^{\circ}$. The magnitude of both the threshold and suprathreshold loss increases slightly for the small field size. Each stimulus was centrally fixated. Vertical error bars represent $\pm 1 S D$. JL has an even pass threshold loss. 


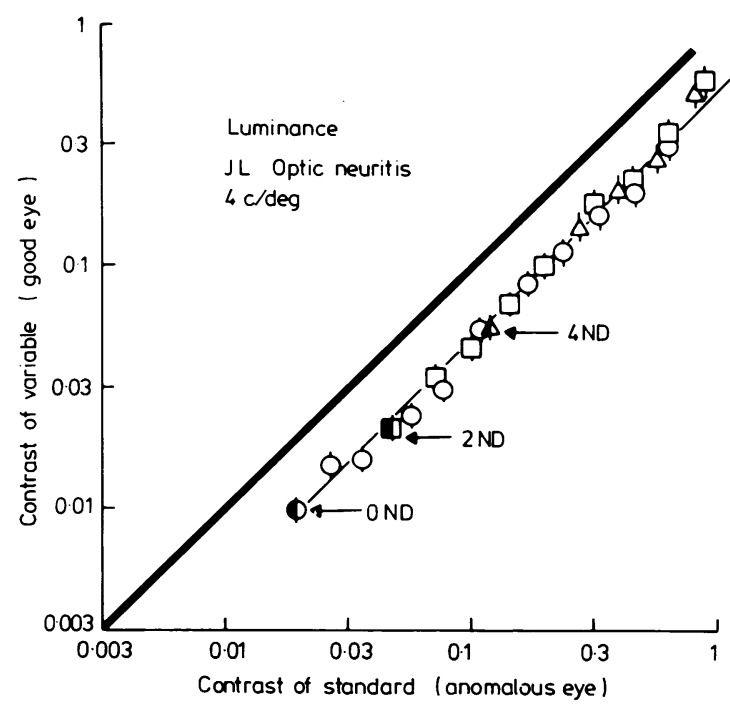

Fig 6 Contrast thresholds (half filled symbols) and suprathreshold matches (open symbols) are displayed for a uniocular case of optic neuritis. Neither the characteristic form of the suprathreshold loss function nor its magnitude depend upon the mean luminance of the stimuli. A parallel displacement of the same magnitude is seen for a wide variation $\left(10^{4}\right)$ of space-averaged luminance. $O N D$ refers to the normal luminance of $200 \mathrm{~cd} / \mathrm{m}^{2}$, whereas $4 \mathrm{ND}$ refers to a luminance of $0.02 \mathrm{~cd} / \mathrm{m}^{2}$. Contrast thresholds for each eye vary with luminance in a systematic but synchronous way. The vertical error bars represent $\pm 1 S D$. This observer displayed only a slight loss of threshold sensitivity.

identical technique to that for contrast discrimination. The results of fig 7 indicate that when only relatively slight deficits occurred for threshold detection no measurable loss in discrimination for incremental (or decremental) suprathreshold stimuli was found. The results for the affected eye and normal fellow eye are similar and were well fitted by a straight line of almost unit slope for $4 \mathrm{c} / \mathrm{deg}$ and slightly less than unity for $0.5 \mathrm{c} / \mathrm{deg}$.

In fig 8 , observer KT had unilateral optic neuritis similar to SS whereas subject IW's condition was bilateral. In this case (IW) the anomalous results are compared with those from an age matched, inexperienced normal observer. Contrast discrimination was anomalous for the affected eyes. The anomalous results were well fit by parallel straight lines of near unit slope, and showed that at all contrast levels the anomalous eye required the same, proportionately larger increment in contrast than normal for correct discrimination. The threshold deficits that are indicated for the normal and anomalous eye with unfilled and filled arrows show little or no corres- pondence with the magnitude of the contrast discrimination abnormality. In the case of SS (fig 7) and KT (fig 8) the contrast matching results displayed a parallel shift at all contrast levels as described earlier. The dashed straight lines in figs 7 and 8 represent the normal discrimination results shifted to lower contrast levels by an amount equal to the contrast matching anomaly (at and above threshold) for the anomalous eye. This dashed line now allows a comparison between normal and anomalous eyes for contrast discriminations about subjectively equivalent base contrasts. For example, if an anomalous (figs 2-5) eye perceives all contrasts a factor of two below their actual level then contrast discrimination thresholds should only be validly compared at equivalent subjective base contrasts. Shifting the normal discrimination results along the $\mathrm{X}$-axis by a factor of two achieves this equivalence of base contrasts.

\section{Discussion}

These matching results suggest that the losses observed for threshold stimuli are just as pronounced at suprathreshold levels. At first glance these results seem to be at odds with the conclusions of Sjostrand and Abrahamsson ${ }^{7}$ who reported that in optic neuritis for mid-high spatial frequencies "the relative contrast loss increased at higher contrast levels". However these authors plotted their results on linear coordinates whereas in the present study they have been plotted on logarithmic coordinates. A closer inspection of their results reveals that the contrast perceived by the anomalous eye is a constant ratio below that of normal in agreement with that found in the present study. Since the perceived contrast varies as the logarithm of the physical contrast (observe fig 3 of Campbell, ${ }^{10}$ where a logarithmic decrease in physical contrast appears to be perceptually linear), this finding is thus interpretable in terms of an abnormality that affects threshold and suprathreshold levels with equal perceptual weight. The assertion by these authors that low spatial frequencies behave differently from mid to high frequencies is not supported by the $0.5 \mathrm{c} / \mathrm{deg}$ results displayed in fig 4 . However, more information is required to answer this point. Thus optic neuritis is one of the only conditions so far described in which there is a true loss of "contrast sensitivity" in its more general sense. Contrast perception for the developmental anomalies of vision (strabismic, anisometropic and deprivation amblyopia) as well as some retinal pathology (Hess unpublished) show striking recruitment above threshold. In these conditions, threshold losses far outweigh those found for suprathreshold stimuli. ${ }^{6}$ This contrast loss in optic 


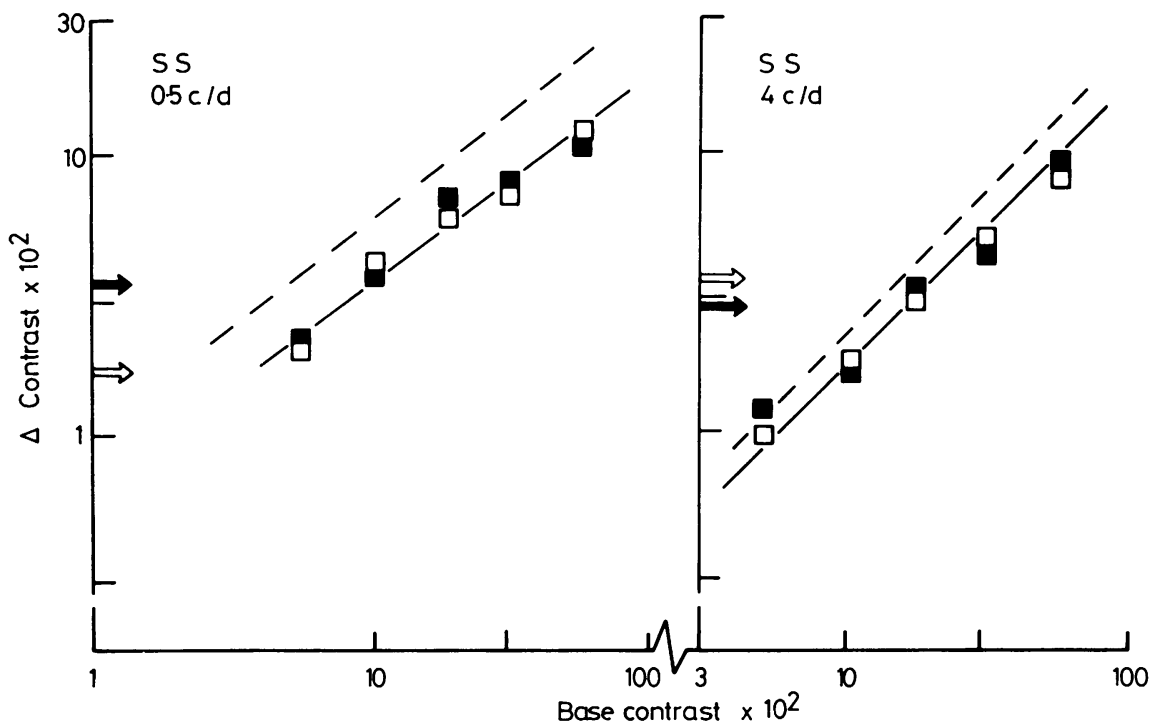

Fig 7 Discrimination results for incremental changes in contrast are displayed for the normal (open symbols) and fellow anomalous eye (filled symbols) of observer SS who exhibited uniocular optic neuritis. Results for two spatial frequencies are displayed. The discrimination results are normal, equal in both eyes and well fitted by a line of unit slope. Filled and unfilled arrows mark the detection thresholds of the anomalous and normal eyes respectively as measured with the same $2 A F C$ procedure. The dashed line represents a parallel displacement of the line best fitting the normal results to lower base contrasts by an amount equal to the difference in contrast matching for the normal and anomalous eyes (see text). Standard deviations are never greater than 1.5 times the symbol size.

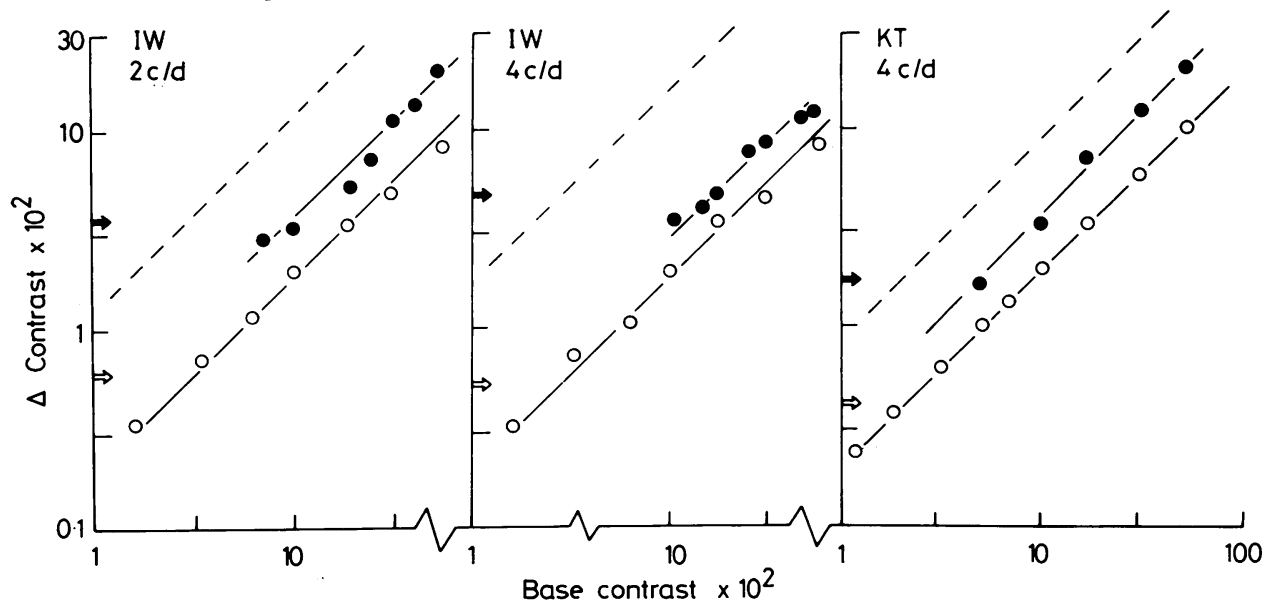

Fig 8 Discrimination results for incremental changes in contrast are displayed for the normal (unfilled symbols) and fellow anomalous eyes (filled symbols) of a case of unilateral optic neuritis (KT) and one of bilateral optic neuritis. In the bilateral case, the results are compared with those from an age matched normal observer (unfilled symbols). The discrimination results are anomalous in all cases and displaced with a constant slope. The filled and unfilled arrows represent the detection thresholds for the anomalous and normal eyes respectively as measured using the same $2 A F C$ procedure. Standard deviations are never greater than 1.5 times the symbol size. The dashed line presents a parallel displacement of the line of best fit to the normal results to lower base contrasts by an amount equal to the difference in contrast matching for the normal and anomalous eye (see text). 
neuritis can be thought of as neural blurring because it is quantitatively similar in its effect at any one spatial frequency (but not necessarily across spatial frequency) to that of optically defocusing the stimulus. This analogy also helps us understand at least some aspects of visual perception of these patients and why in particular they report that their vision appears "washed out", "faded" or "blurred".

It is interesting to note that the form of the contrast loss does not depend on field size or luminance. Hess and Plant ${ }^{11}$ have found that the effect of temporal frequency variation on threshold losses in optic neuritis, can be mimmicked in normal observers by artificial scotomata. They have suggested that (as seems likely from the pathology) patchy involvement of the optic nerve, and hence visual field, might result in islands of relatively preserved function which would account for their findings. The present results suggest that the effect of this patchy disturbance on perception does not vary with contrast or mean luminance and does not vary greatly with eccentricity. There is, however, some variation with field size as would be predicted from a non homogeneous disorder (fig 7). In other words, those abnormal regions of the visual field are characterised by a reduced gain (divisive loss) such that they represent absolute scotomata for threshold test stimuli and relative scotomata for suprathreshold test stimuli.

The contrast discrimination results are only slightly anomalous compared with the steady rate anomaly seen from matching experiments. The contrast discrimination anomalies in optic neuritis are not simply explained by the loss of subjective contrast that has already been described in the first section of this paper. For example, the contrast discrimination results of normal eyes and those with optic neuritis do not match even when compared at subjectively equivalent base contrasts (for example, by displacing the normal discrimination function to lower base contrasts by an amount equal to the loss of perceived contrast in optic neuritis). These results suggest that either contrast discrimination is better than normal in optic neuritis or the adjustment to equate base contrast in terms of their subjective appearance is invalid. The latter suggestion seems the more likely. This in turn implies that matching and discrimination have quite different and independent physiological substrates, a conclusion also seen in other results ${ }^{1112}$ in which contrast discrimination in the recruiting region of amblyopic contrast matching functions is never better than normal.

Neither of the two models of contrast coding for normal vision are suitable for the description of these results in optic neuritis. Kulikowski ${ }^{5}$ proposed a model in which the subjective contrast (Cs) was directly proportional to the physical contrast (C) minus the contrast threshold $\left(\mathrm{K}_{\mathrm{D}}\right)$. That is: Cs $\propto$ log $\mathrm{C}-\mathrm{K}_{\mathrm{D}}$. Georgeson and Sullivan, ${ }^{4}$ on the other hand, postulated that subjective contrast for stimuli having different thresholds was equated above a criterion value, that is subjective and physical contrasts are related as follows: if $\mathrm{C}<0.4$ then $\mathrm{Cs}=\log$ C.

The present results however, are well described by a divisive relationship that is: $C s=\log C / K_{R}$, where $K_{R}$ is the ratio of contrast thresholds.

In an attempt to explain the contrast recruiting responses that typify contrast perception for the developmental anomalies of vision (amblyopia) an alternative proposition ${ }^{12}$ has been proposed to that of Georgeson and Sullivan ${ }^{4}$ or Kulikowski. ${ }^{5}$ The suggestion involves the notion of more than one contrast "channel". In its simplest form two mechanisms, one responding to lower contrasts (more sensitive) than the other. Such a model which has some neurophysiological support in the findings of Shapley, Kaplan and Soodak ${ }^{13}$ and Lennie and Derrington (personal communication) can account for the numerous types of threshold-suprathreshold relationship that have been so far reported for normal and anomalous vision. Following on from this idea the present results suggest that, unlike amblyopia where the more sensitive contrast channel may be selectively affected, ${ }^{12}$ in optic neuritis all contrast channels may be equally affected.

Optic neuritis thus represents a condition in which no suprathreshold recruitment in contrast sensation occurs; contrast thresholds and suprathreshold perception are equally affected. In this way the processing of contrast by the visual system may have something in common with the processing of loudness by the auditory system. In audition, anomalies of the inner ear exhibit loudness recruitment. Analogously, the various amblyopias of vision exhibit contrast recruitment. In audition, pathology to the auditory nerve does not result in loudness recruitment; thresholds and suprathreshold perception being equally affected or sometimes suprathreshold perception being more greatly affected. ${ }^{14}$ In vision, the present results suggest that pathology to the optic nerve results in analogous situation for contrast (no recruitment).

I acknowledge the support obtained from the Medical Research Council of Great Britain and the Wellcome Trust. I am grateful to Clive Hood for technical assistance and to Brian Moore, Gordon Plant, Jonathan Pointer, Robert Weale, David Tolhurst and Mark Georgeson for suggestions and useful discussions. I am specially grateful to Fergus Campbell for his encouragement and advice. 


\section{References}

${ }^{1}$ Bodis-Wollner I. Visual acuity and contrast sensitivity in patients with cerebral lesions. Science 1972; 178:769-71.

${ }^{2}$ Regan D, Silver R, Murray TJ. Visual acuity and contrast sensitivity in multiple sclerosis: hidden visual loss. Brain 1977;100:563.

${ }^{3}$ Zimmern RL, Campbell FW, Wilkinson IMSW. Subtle disturbances of vision after optic neuritis elicited by studying contrast sensitivity. J Neurol Neurosurg Psychiatry 1979;42:407-12.

${ }^{4}$ Georgeson M, Sullivan G. Contrast constancy: Deblurring in human vision by spatial frequency channels. $J$ Physiol (Lond) 1975;252:627-56.

${ }^{5}$ Kulikowski JJ. Effective contrast constancy. Vision Res 1976;16:1419-31.

${ }^{6}$ Hess RF, Bradley A, Piotrowski L. Contrast coding in amblyopia: I Differences in the neural basis of human amblyopia. Proc Roy Soc B 1983;217:309-30.

${ }^{7}$ Sjostrand J, Abrahamsson M. Suprathreshold vision in acute optic neuritis. J Neurol Neurosurg Psychiatry 1982;45:227-34.
${ }^{8}$ Blakemore C, Campbell FW. On the existence of neurones in the human visual system selectively sensitive to the orientation and size of retinal images. $J$ Physiol (Lond) 1969;203:237-60.

9 Watson AB, Pelli D. The Quest psychophysical procedure. Appl Vision Assoc Newsletter 1979;14:6-7.

${ }^{10}$ Campbell FW. The eye as an optical filter. Proc IEEE 1968;56:1009-14.

$"$ Hess RF, Plant G. The effect of temporal frequency variation on contrast threshold deficits in optic neuritis. $J$ Neurol Neurosurg Psychiatry 1983;46:322-30.

12 Hess RF. Contrast coding in amblyopia: II Physiological basis of contrast recruitment. Proc Roy Soc B 1983;217:331-40.

${ }^{13}$ Shapley R, Kaplan E, Soodak R. Spatial summation and contrast sensitivity of $x$ and $y$ cells in the lateral geniculate nucleus of the macaque. Nature 1981;292:543-5.

14 Dix MR, Hallpike CS, Hood JD. Observation upon the loudness recruitment phenomenon, with special reference to the differential diagnosis of disorders of the inner ear and VIII nerve. Proc Roy Soc Med 1948;41:516-26. 